\title{
SUSY searches in ATLAS and CMS
}

\author{
Vasiliki A. Mitsou* on behalf of the ATLAS and CMS Collaborations \\ Instituto de Física Corpuscular (IFIC), CSIC - Universitat de València, \\ C/ Catedrático José Beltrán 2, E-46980 Paterna (Valencia), Spain \\ E-mail: vasiliki.mitsou@ific.uv.es
}

Supersymmetry (SUSY) is one of the most relevant scenarios of new physics searched for at the CERN Large Hadron Collider (LHC). In this contribution, the principal search strategies employed by the ATLAS and CMS experiments are outlined and the most recent results for analyses targeting SUSY discovery are presented. A wide range of signatures are covered motivated by various theoretical scenarios and topologies: strong production, third-generation fermions, electroweak production, $R$-parity violation and long-lived particles. Most results presented here are based on the full Run 2 dataset at $\sqrt{s}=13 \mathrm{TeV}$ centre-of-mass energy by each experiment at the LHC.

Corfu Summer Institute 2019 "School and Workshops on Elementary Particle Physics and Gravity" (CORFU2019)

31 August - 25 September 2019

Corfu, Greece

*Speaker. 


\section{Introduction}

Supersymmetry (SUSY) [1 -4] is an extension of the Standard Model (SM) which assigns to each SM field a superpartner field with a spin differing by a half unit. SUSY provides elegant solutions to several open issues in the SM, such as the hierarchy problem, the identity of dark matter, and grand unification.

SUSY searches in collider experiments typically focus on events with high transverse missing energy $\left(E_{\mathrm{T}}^{\mathrm{miss}}\right)$ which can arise from the (weakly interacting) lightest supersymmetric particle (LSP), in the case of $R$-parity conserving (RPC) SUSY, or from neutrinos produced in LSP decays, when $R$-parity is violated (RPV). Hence, the event selection criteria of inclusive channels are based on large $E_{\mathrm{T}}^{\text {miss }}$, no or few light leptons $(e, \mu)$, many jets and/or $b$-jets, $\tau$-leptons and photons. The exact sets of cuts (signal regions, SRs) are a compromise between the necessity to suppress events coming from known SM processes while maintaining a statistically sufficient number of surviving SUSY events. Typical SM backgrounds are top-quark production — including single-top - $W / Z$ in association with jets, dibosons and QCD multi-jet events. These are estimated using semi- or fully data-driven techniques in so-called control regions (CRs). Before unblinding the SRs, the background estimation is validated in validation regions (VRs), which lie between the defined SRs and CRs. Less dominant backgrounds are estimated by Monte Carlo (MC) simulation. Although the various analyses are motivated and optimised for a specific SUSY scenario or topology, the interpretation of the results may be extended to various SUSY models, hence both collaborations are investing huge effort to facilitate the reinterpretation of their search results [5].

A summary of recent results (as of September 2019) on searches for SUSY with and without $R$-parity conservation is presented. The majority of the reported results are based on up to $\sim 140 \mathrm{fb}^{-1}$ of data from $p p$ collisions at a centre-of-mass energy of $\sqrt{s}=13 \mathrm{TeV}$ recorded by ATLAS [6] and CMS [7] during Run 2 (2015-2018) at the Large Hadron Collider (LHC) [8]. Analyses exploring $R$-parity conserving SUSY models are currently divided into inclusive searches for: (a) squarks and gluinos, (b) third-generation fermions, and (c) electroweak production $\left(\tilde{\chi}^{0}, \tilde{\chi}^{ \pm}, \tilde{\ell}\right)$. Recent results from each category of ATLAS searches are presented in Sections 2, 3 and 4, respectively. Searches designed to look for models with broken $R$-parity are discussed in Section 5 , while in Section 6 recent developments on the long-lived-sparticle front are presented. Lastly, Section 7 summarises the results presented here.

\section{Squarks and gluinos}

The production of the supersymmetric partners of quarks and gluons (squarks and gluinos) is characterised by large cross-sections at the LHC. Strong SUSY production is searched in events with large jet multiplicities and high missing transverse momentum, with and without leptons. Various channels fall into this class of searches; here two cases are showcased: the fully hadronic channel and the final state with two-same sign or three leptons.

\subsection{Fully hadronic channel}

In the 0-lepton analyses, selection strategies to search for squarks and gluinos in final states containing several jets, missing transverse momentum but no electrons or muons are presented. 
Both collaborations have developed and utilised new techniques and novel observables with respect to earlier searches $[9,10]$.

In the first search reported here [11], CMS used a sample of $p p$ collisions corresponding to an integrated luminosity of $137 \mathrm{fb}^{-1}$, representing essentially the full LHC Run 2 data sample. The analysis was performed in 174 nonoverlapping search bins in a four-dimensional search region defined in terms of the number of jets, the number of $b$-tagged jets, the scalar sum of jet transverse momenta, $H_{\mathrm{T}}$, and the magnitude of the vector sum of jet transverse momenta, $H_{\mathrm{T}}^{\text {miss }}$. The events were required to satisfy $H_{\mathrm{T}}^{\text {miss }}>300 \mathrm{GeV}, H_{\mathrm{T}}>300 \mathrm{GeV}$, and to have at least two jets with transverse momentum $p_{\mathrm{T}}>30 \mathrm{GeV}$. Events with isolated high- $p_{\mathrm{T}}$ leptons or photons were vetoed.

The principal backgrounds arise from events with neutrino production or jet mismeasurement. The SM background was evaluated using control regions in data supplemented by information from MC event simulation [11]. The $H_{\mathrm{T}}^{\text {miss }}$ distributions of Figure 1 depict a comparison between the background predictions, the data and two example signal scenarios, one with $\Delta m \gg 0$ and the other with $\Delta m \simeq 0$, where $\Delta m$ is the difference between the $\tilde{g}$ or $\tilde{q}$ mass and the sum of the masses of the particles into which it decays.

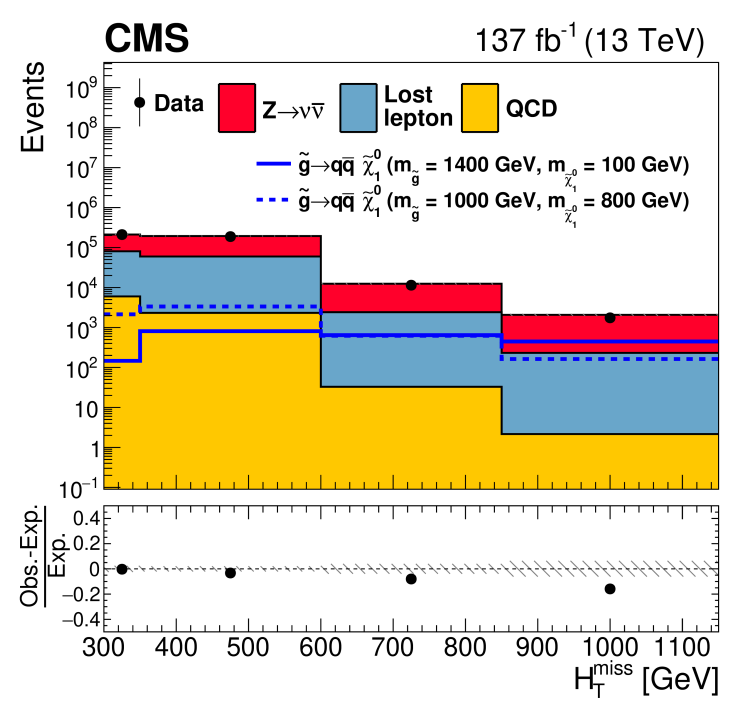

Figure 1: One-dimensional projection of the data and pre-fit $\mathrm{SM}$ predictions in $H_{\mathrm{T}}^{\mathrm{miss}}$. The hatched regions indicate the total uncertainties in the background predictions. The distributions for two example signal scenarios are also shown. From [11].

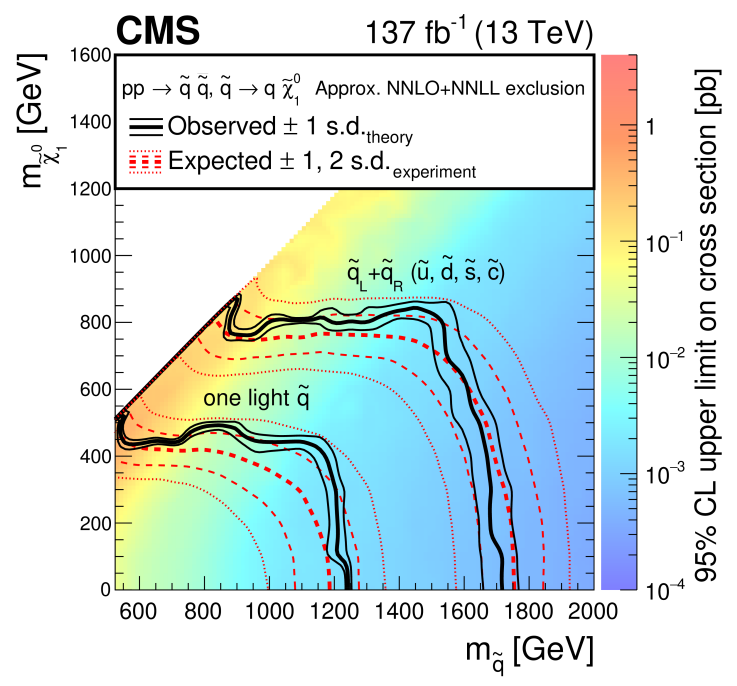

Figure 2: 95\% CL exclusion limits for light-flavour $\tilde{q} \tilde{q}$ production using $M_{\mathrm{T} 2}$. The thick (thin) black curves show the observed limits (uncertainties), while the dashed red lines indicate the expected limits with $\pm 1 \sigma$ and $\pm 2 \sigma$ ranges. From [12].

No significant excess in the event yield was observed relative to the expected background contributions from SM processes. Limits on the pair production of gluinos and squarks were obtained in the framework of simplified models for sparticle production and decay processes. Assuming the LSP to be the $\tilde{\chi}_{1}^{0}$, lower limits on the $\tilde{g}$ mass as large as 2000 to $2310 \mathrm{GeV}$ were obtained at $95 \%$ confidence level (CL), while lower limits on the $\tilde{q}$ mass as large as 1190 to $1630 \mathrm{GeV}$ were set, depending on the production scenario [11].

Another inclusive search has been performed by CMS targeting SUSY and other beyond-theSM (BSM) scenarios, based on signal regions defined by the hadronic energy in the event, the jet 
multiplicity, the number of $b$-jets, and the value of the kinematic variable $M_{\mathrm{T} 2}$ [13] for events with at least two jets [12]. For events with exactly one jet, the jet $p_{\mathrm{T}}$ is used instead. The $M_{\mathrm{T} 2}$ variable quantifies the transverse momentum imbalance of the event.

No excess event yield was observed above the predicted SM background. A range of BSM models have been constrained, with the pair production of gluinos and squarks in the context of SUSY conserving $R$-parity being among them. As an example, the exclusion limits for light-flavour squark pair production are shown in Figure 2 for two assumptions: one light $\tilde{q}$ or the sum from all light $\tilde{q}_{\mathrm{L}}$ and $\tilde{q}_{\mathrm{R}}$. This search probed $\tilde{g}$ masses up to $2250 \mathrm{GeV}$ and $\tilde{\chi}_{1}^{0}$ masses up to $1525 \mathrm{GeV}$, as well as light-flavour, bottom, and top squark masses up to 1710,1240 , and $1200 \mathrm{GeV}$, respectively, and $\tilde{\chi}_{1}^{0}$ masses up to 870,700 , and $580 \mathrm{GeV}$ in each respective scenario [12].

ATLAS released results from a fully hadronic search [14] based on $139 \mathrm{fb}^{-1}$, representing the full Run 2 dataset. Two approaches were followed. The first was based on a multiple-bin analysis in the effective mass, $m_{\mathrm{eff}}$, defined as the scalar sum of $E_{\mathrm{T}}^{\text {miss }}$ and the $p_{\mathrm{T}}$ of all jets with $p_{\mathrm{T}}>50 \mathrm{GeV}$, extending the previous ATLAS search [9]. Among other preselection cuts, events were required to have $E_{\mathrm{T}}^{\text {miss }}>300 \mathrm{GeV}$ and $m_{\mathrm{eff}}>800 \mathrm{GeV}$. The second approach, referred to as the BDT search, was a complementary search which used boosted decision trees (BDTs) implemented in the TMVA framework [15] for the event selection. The BDT search improved the sensitivity to models where gluinos decay via an intermediate chargino because of its highly optimised design and ability to exploit the correlations between variables.

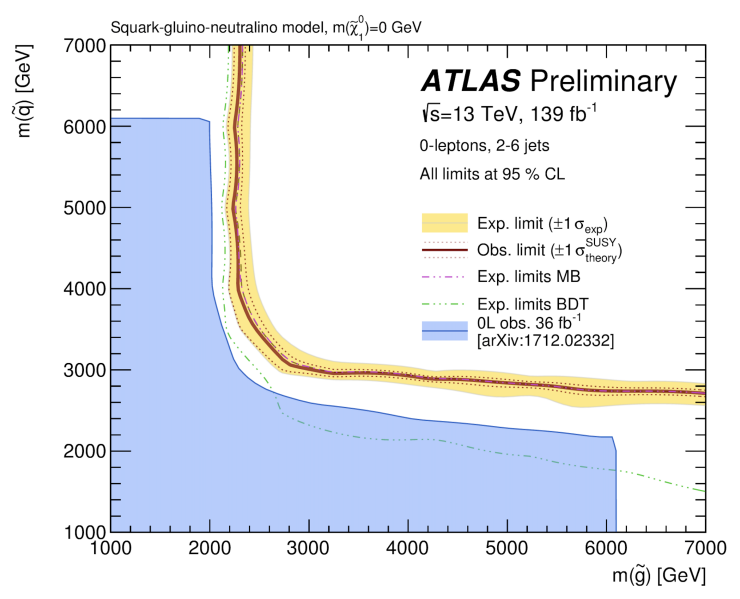

Figure 3: Exclusion limits for the combined $\tilde{q} \tilde{q}$, $\tilde{g} \tilde{g}$ and $\tilde{q} \tilde{g}$ production, assuming a massless purely bino $\tilde{\chi}_{1}^{0}$. Expected limits from the multi-bin (MB) and BDT searches are also shown, with the yellow bands indicating the $\pm 1 \sigma$ range of the expected fluctuations due to experimental and backgroundonly theoretical uncertainties. Observed limits are indicated by maroon curves where the solid (dotted) contour represents the nominal limit (signal cross-section theoretical uncertainties). From [14].

No significant deviation from the background expectation was found. Results were interpreted in terms of simplified models with only first- and second-generation squarks, or gluinos, together with a $\tilde{\chi}_{1}^{0}$ LSP, with the masses of all other SUSY particles effectively decoupled. For a massless $\tilde{\chi}_{1}^{0}$, gluino masses below $2.35 \mathrm{TeV}$ were excluded at $95 \% \mathrm{CL}$ in a simplified model with only gluinos and the lightest neutralino [14]. For a simplified model involving the strong production of squarks of the first and second generations, with decays to a massless $\tilde{\chi}_{1}^{0}, \tilde{q}$ masses below $1.94 \mathrm{TeV}$ were excluded, assuming mass-degenerate squarks of the first two generations. In models with $\tilde{q} \tilde{q}$ and $\tilde{g} \tilde{g}$, each decaying via an intermediate $\tilde{\chi}^{ \pm}$to one quark or two quarks, a $W$ boson and a $\tilde{\chi}_{1}^{0}, \tilde{q}$ masses below $1.59 \mathrm{TeV}$ and $\tilde{g}$ masses below $2.19 \mathrm{TeV}$ were excluded for massless $\tilde{\chi}_{1}^{0}$. As shown in Figure 3 , in models with combined production of squark pairs, gluino pairs, and of squark-gluino pairs, a lower limit of $3000 \mathrm{GeV}$ for equal $\tilde{q}$ and $\tilde{g}$ mass was found for the scenario with a massless 
$\tilde{\chi}_{1}^{0}$. These results substantially extended the region of supersymmetric parameter space previously excluded by similar ATLAS searches [9], also shown in Figure 3.

\subsection{Two same-sign or three leptons}

In the SM, the production of multiple jets in conjunction with two same-sign (SS) or three or more charged leptons is a very rare process in proton-proton collisions. Therefore, these final states provide a promising starting point in the search for BSM physics. In SUSY models, for instance, the decay chain of pair-produced gluinos or squarks can contain multiple $W$ or $Z$ bosons, possibly including one or several pairs of SS $W$ bosons. Such a decay chain is realised, for example in $\tilde{g}$ pair production, when a $\tilde{g}$ decays into a $t \bar{t}$ and a $\tilde{\chi}_{1}^{0}$, the T1ttt model. The results of such an analysis [16] conducted by CMS with $137 \mathrm{fb}^{-1}$ is shown in Figure 4 in the $m_{\tilde{\chi}_{1}^{0}}$ vs. $m_{\tilde{g}}$ plane. The lower mass limits are as large as $2.1 \mathrm{TeV}$ for gluinos (when it decays via $\tilde{g} \rightarrow t b s$ through an RPV $\lambda^{\prime \prime}$ coupling) and $0.9 \mathrm{TeV}$ for top and bottom squarks, depending on the details of the model. The results extended the $\tilde{g}$ and $\tilde{q}$ mass observed and expected exclusions by up to $200 \mathrm{GeV}$ compared to the previous versions of this analysis $[17,18]$.

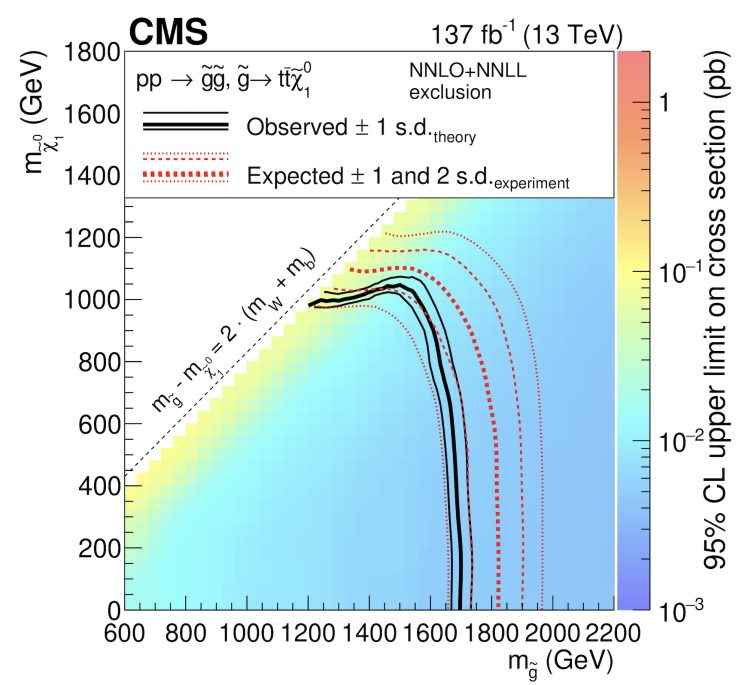

Figure 4: 95\% CL exclusion region for the T1tttt model. The colour scale shows the excluded crosssection values. The solid black thick (thin) curve shows the observed exclusion limit (its $\pm 1 \sigma$ variations). The dashed red curves show the expected limits with $\pm 1 \sigma$ and $\pm 2 \sigma$ uncertainties. From [16].

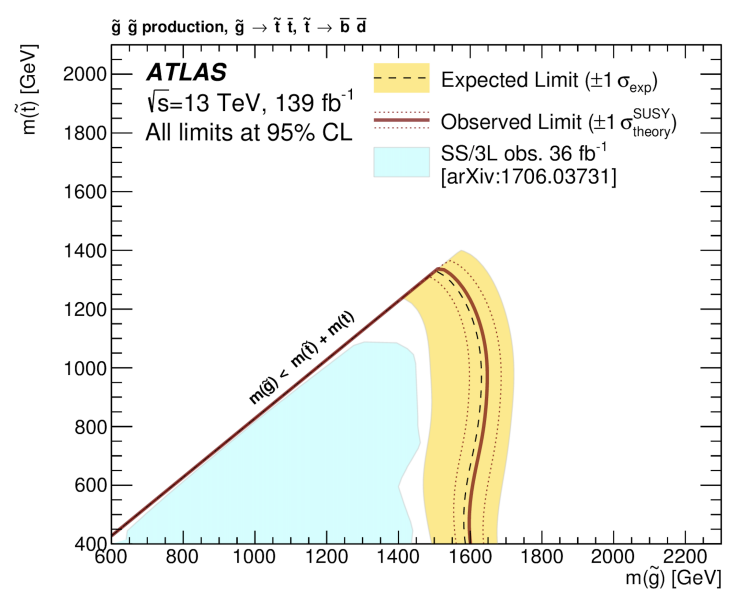

Figure 5: 95\% CL exclusion limits on $\tilde{g} \tilde{g}$ production with a decay chain involving an RPV $\lambda_{313}^{\prime \prime}$ coupling. The yellow band displays the $\pm 1 \sigma$ range of the expected fluctuations around the mean expected limit. The signal process cross-section uncertainties in are represented by the dashed lines around the observed limits. The reach of the previous analysis [19] is also shown. From [20].

In the ATLAS search [20], also using the full Run 2 dataset, five signal regions were defined to cover a broad range of supersymmetric process signatures, assuming $R$-parity conservation or violation. With no significant excess over the SM prediction, results were interpreted in the framework of four simplified models featuring pair production of gluinos or third-generation squarks. Lower limits on particle masses were derived at 95\% CL for these models, reaching up to $1.6 \mathrm{TeV}$ for gluinos and $750 \mathrm{GeV}$ for bottom and top squarks, significantly extending the previous exclusion limits obtained with a similar search [19] for a smaller dataset. An example of limits for an RPV 
simplified model of $\tilde{g}$ pair production and $\tilde{g} \rightarrow \tilde{t} t$ with $\tilde{t} \rightarrow b d$ is shown in Figure 5, obtained with the (RPV optimised) Rpv2L SR. Model-independent limits on the cross-section of possible BSM signal contributions to the SRs were also reported [20].

\section{Third-generation squarks}

The mixing of left- and right-handed gauge states which provides the mass eigenstates of the scalar quarks and leptons can lead to relatively light $3^{\text {rd }}$ generation particles. Stop $\left(\tilde{t}_{1}\right)$ and sbottom $\left(\tilde{b}_{1}\right)$ with a sub-TeV mass are favoured by the naturalness argument [21, 22], while the stau $\left(\tilde{\tau}_{1}\right)$ is the lightest $\tilde{\ell}$ in many models. Therefore, these sparticles could be abundantly produced either directly or through $\tilde{g}$ production and subsequent decay. Such events are characterised by several energetic jets (some of them $b$-jets), possibly accompanied by light leptons, as well as large $E_{\mathrm{T}}^{\text {miss }}$.

\subsection{Stop pair production}

CMS searched for the $\tilde{t}$ in final states with one isolated electron or muon, jets and high $E_{\mathrm{T}}^{\text {miss }}$ using $137 \mathrm{fb}^{-1}$ of $p p$ collision data at $\sqrt{s}=13 \mathrm{TeV}$ [23]. One of the variables used in the analysis is a modified version of the topness variable [24], $t_{\text {mod }}$, defined in Ref. [25]. The $t_{\bmod }$ variable is a $\chi^{2}-$ like variable that discriminates signal from leptonically decaying $t \bar{t}$ events: an event with a small value of $t_{\text {mod }}$ is likely to be a dilepton $t \bar{t}$ event, while signal events tend to have larger $t_{\text {mod }}$ values. This is illustrated in Figure 6, where $t_{\text {mod }}$ distributions are shown after the preselection requirements for SM background processes, obtained with MC simulation, and three signal scenarios.

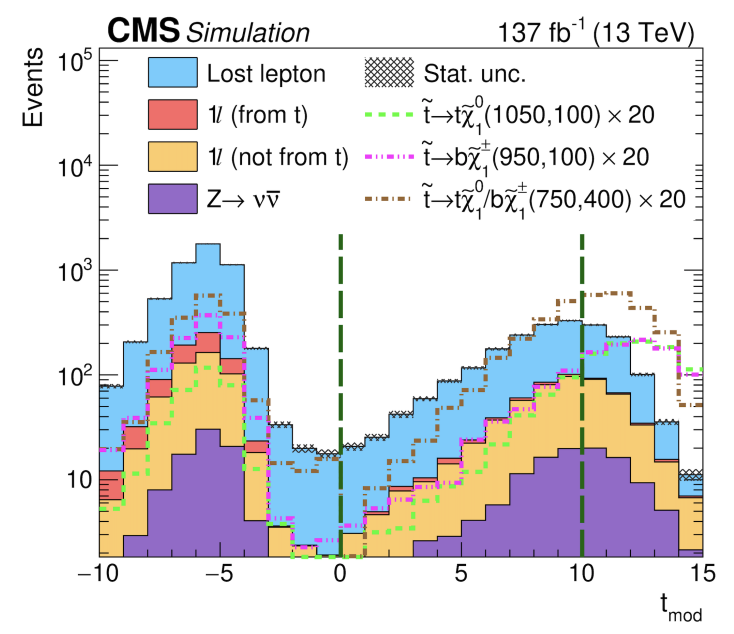

Figure 6: $t_{\text {mod }}$ distributions after preselection requirements for SM background and three signal hypotheses; the numbers in the legends refer to $m_{\tilde{t}}$ and $m_{\tilde{\chi}_{1}^{0}}$, respectively. For models with $b \tilde{\chi}_{1}^{ \pm}$decays, $m_{\tilde{\chi}_{1}^{ \pm}}=\left(m_{\tilde{t}}+m_{\tilde{\chi}_{1}^{0}}\right) / 2$. The green, dashed vertical lines mark the locations of the binning or tagging requirements. From [23].

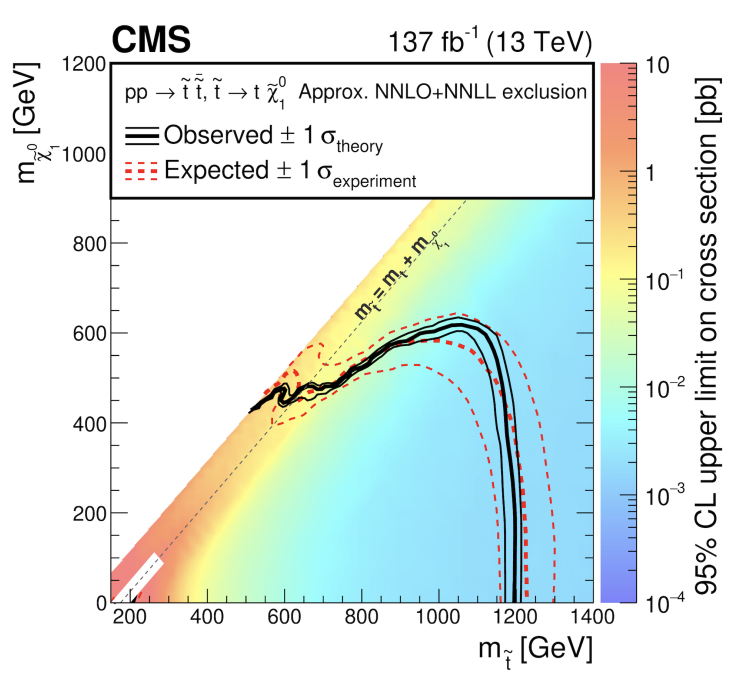

Figure 7: $95 \%$ CL exclusion limits for $\tilde{t} \bar{t} \rightarrow t \bar{t} \tilde{\chi}_{1}^{0} \tilde{\chi}_{1}^{0}$. The coloured map shows the upper limits on the production cross-section. The thick (thin) black curve shows the observed exclusion region (its theoretical uncertainties). The red curves show the expected exclusion and its uncertainties. From [23].

The observed data were consistent with the predicted SM background processes. Exclusions were set in the context of simplified top squark pair production models. For models with a $\tilde{t}$ mass 
of $1 \mathrm{TeV}, \tilde{\chi}_{1}^{0}$ masses up to $600 \mathrm{GeV}$ were excluded [23]. Depending on the model, exclusion limits at $95 \% \mathrm{CL}$ for $\tilde{t}$ masses up to $1.2 \mathrm{TeV}$ were set for a massless $\tilde{\chi}_{1}^{0}$, as shown in Figure 7 . The white band excluded from the limits corresponds to the region $\left|m_{\tilde{t}}-m_{t}-m_{\tilde{\chi}_{1}^{0}}\right|<25 \mathrm{GeV}, m_{\tilde{t}}<275 \mathrm{GeV}$, where the selection acceptance for top squark pair production changes rapidly and is therefore very sensitive to the details of the simulation.

\subsection{Sbottom pair production}

The ATLAS experiment has searched for the $\tilde{b}_{1}$ pair production using $139 \mathrm{fb}^{-1}$ of $p p$ data at $13 \mathrm{TeV}$ [26]. In the SUSY scenarios considered both bottom squarks decay via $\tilde{b}_{1} \rightarrow b \tilde{\chi}_{2}^{0}$. Each $\tilde{\chi}_{2}^{0}$ is assumed to subsequently decay with $100 \%$ branching ratio into a Higgs boson and the $\tilde{\chi}_{1}^{0}$ LSP: $\tilde{\chi}_{2}^{0} \rightarrow h \tilde{\chi}_{1}^{0}$. The first signal mass configuration targeted has a constant LSP mass of $60 \mathrm{GeV}$ motivated by dark-matter relic density measurements and might be favoured in Higgs-pole annihilation scenarios [27], where $m_{\tilde{\chi}_{1}^{0}}=m_{h} / 2$. The second scenario has a constant mass difference $m_{\tilde{\chi}_{2}^{0}}-m_{\tilde{\chi}_{1}^{0}}=130 \mathrm{GeV}$, sufficient to produce an on-shell Higgs boson.

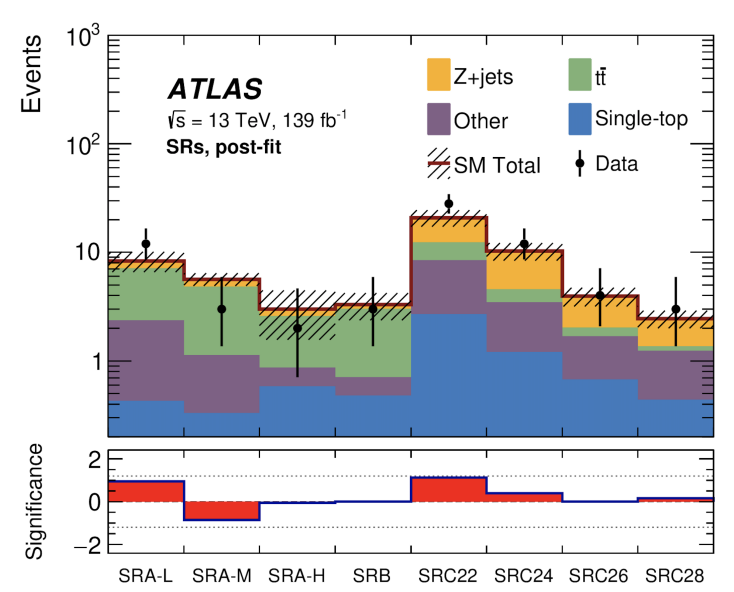

Figure 8: Results of the background-only fit to the CRs extrapolated to all SRs. The upper (lower) panel shows data and background (significance) in each SR. The label "Other" includes diboson, $W+$ jets and $t \bar{t}+W / Z / h$ backgrounds. From [26].

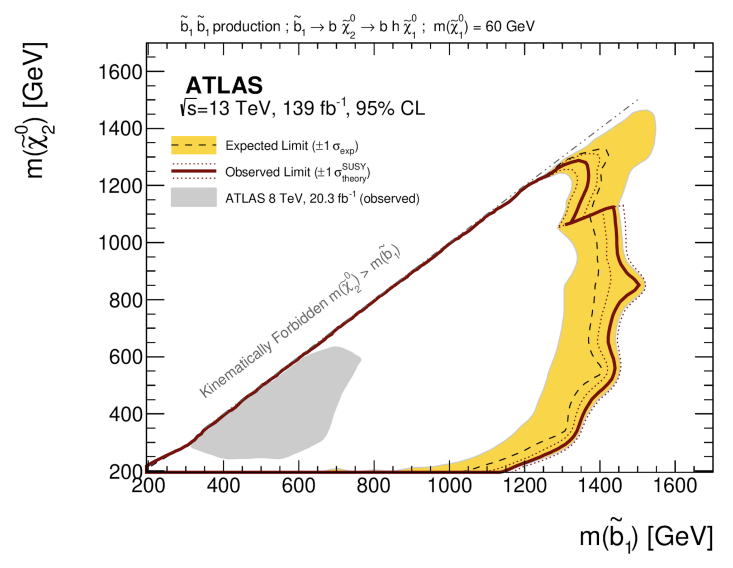

Figure 9: Exclusion contour at the $95 \% \mathrm{CL}$ in the $\left(\tilde{b}_{1}, \tilde{\chi}_{2}^{0}\right)$ mass parameter space for the $m_{\tilde{\chi}_{1}^{0}}=$ $60 \mathrm{GeV}$ signal scenario. The theory uncertainty band contains the systematic uncertainties on the signal model under consideration and the uncertainty in the signal cross-section. From [26].

The final states considered contain no charged leptons, three or more $b$-jets, and large $E_{\mathrm{T}}^{\text {miss }}$. Three sets of non-orthogonal SRs were defined to target different mass hierarchies of the SUSY particles involved. These definitions exploited various discriminating observables and algorithms developed to explicitly reconstruct Higgs boson candidates in the decay chain. As shown in Figure 8, no significant excess of events over the SM background expectation was observed in any of the signal regions considered. Limits at the 95\% CL were placed in the supersymmetric models considered, and bottom-squarks with mass up to $1.5 \mathrm{TeV}$ were excluded [26]. As illustrated in Figure 9, the limits for the $m_{\tilde{\chi}_{1}^{0}}=60 \mathrm{GeV}$ scenario extend considerably further than those from an earlier similar analysis [28]. 


\section{Electroweak gaugino and slepton production}

Electroweak SUSY production is motivated by naturalness [21,22] and it is complementary to searches focusing on strongly produced sparticles. Natural models of SUSY favour light chargino and neutralino masses, in a range well accessible at the LHC. In the event that the strong production is sufficiently suppressed due to heavy squarks and gluinos, electroweak (EW) SUSY production may be the dominant SUSY production mechanism at the LHC.

\subsection{Electroweakinos with $h \rightarrow \gamma \gamma$}

Two analyses are presented here, one from each experiment, CMS and ATLAS, which target weak gaugino or electroweakino $\left(\tilde{\chi}^{0}\right.$ and $\left.\tilde{\chi}^{ \pm}\right)$production with at least one Higgs boson being produced and decaying to two photons. The presence of charged leptons, additional Higgs boson candidates, and various kinematic variables were used to categorise events into search regions that are sensitive to different SUSY scenarios. The CMS results [29] were based on $p p$ data at $13 \mathrm{TeV}$ corresponding to $77.5 \mathrm{fb}^{-1}$. Photon pairs in the central region of the detector were used to reconstruct Higgs boson candidates in their invariant mass, $m_{\gamma \gamma}$, distribution, as depicted in Figure 10. Charged leptons and $b$-jets were used to identify the decay products of an additional boson.

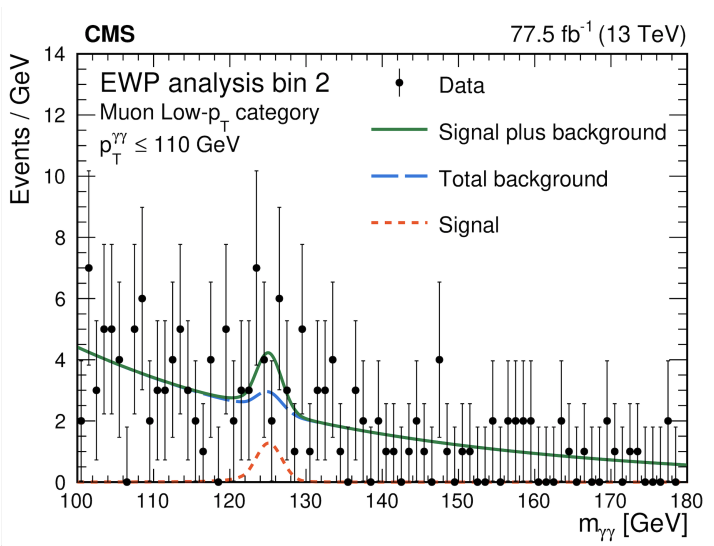

Figure 10: The diphoton mass distribution for an example search bin with the signal-plus-background fit. From [29].

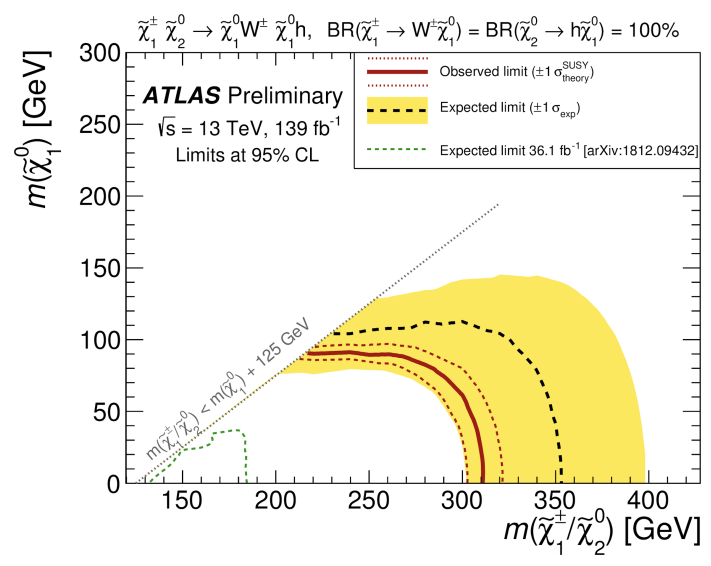

Figure 11: 95\% CL observed (red solid line) and expected (black dashed line) exclusion limits for the $\tilde{\chi}_{1}^{ \pm} \tilde{\chi}_{2}^{0}$ production. From [30].

No statistically significant excess of events was observed relative to the SM expectations, hence CMS excluded wino-like $\tilde{\chi}_{1}^{ \pm} \tilde{\chi}_{2}^{0}$ production for $m_{\tilde{\chi}_{1}^{ \pm}}=m_{\tilde{\chi}_{2}^{0}}<235 \mathrm{GeV}$ with a $m_{\tilde{G}}=1 \mathrm{GeV}$ and higgsino-like $\tilde{\chi}_{1}^{ \pm} \tilde{\chi}_{2}^{0}$ production in the case where the $\tilde{\chi}_{1}^{0}$ decays exclusively to a Higgs boson and a gravitino $\tilde{G}$ for $m_{\tilde{\chi}_{1}^{0}}<290 \mathrm{GeV}[29]$.

The ATLAS study was based on the full Run 2 dataset of $139 \mathrm{fb}^{-1}$ [30]. No significant excess over the expected background was observed. Upper limits at 95\% CL for a massless $\tilde{\chi}_{1}^{0}$ were set on several electroweakino production cross-sections and on the visible cross-section for other BSM physics processes. In the context of simplified SUSY models, 95\% CL limits of up to $315 \mathrm{GeV}$ in $m_{\tilde{\chi}_{1}^{ \pm} / \tilde{\chi}_{2}^{0}}$, where $m_{\tilde{\chi}_{1}^{0}}=0.5 \mathrm{GeV}$, were set. Limits at $95 \% \mathrm{CL}$ were also set on the $\tilde{\chi}_{1}^{ \pm} \tilde{\chi}_{2}^{0}$ cross-section 
in the mass plane of $m_{\tilde{\chi}_{1}^{ \pm} / \tilde{\chi}_{2}^{0}}$ vs. $m_{\tilde{\chi}_{1}^{0}}$, as shown in Figure 11, that extended significantly previous results from the $36.1 \mathrm{fb}^{-1}$ analysis [31].

\subsection{Two leptons and large $E_{\mathrm{T}}^{\mathrm{miss}}$}

ATLAS carried out a search [32] for the EW production of charginos and sleptons decaying into final states with exactly two oppositely charged leptons and large $E_{\mathrm{T}}^{\text {miss }}$ with $139 \mathrm{fb}^{-1}$ of $p p$ data. SRs vetoing or requiring exactly one energetic jet were defined. Three scenarios were considered: the production of $\tilde{\chi}_{1}^{ \pm} \tilde{\chi}_{1}^{\mp}$, followed by their decays into final states with leptons and the $\tilde{\chi}_{1}^{0}$ via either $W$ bosons (cf. Figure 12, left) or $\tilde{\ell} / \tilde{v}$, and the direct production of $\tilde{\ell}$ pairs, where each $\tilde{\ell}$ decays directly into the $\tilde{\chi}_{1}^{0}$ and a lepton. No significant deviations from the SM expectations were observed and limits at 95\% CL were set on the masses of relevant supersymmetric particles in each of these scenarios. For a massless $\tilde{\chi}_{1}^{0}$, masses up to $420 \mathrm{GeV}$ were excluded for the production of the $\tilde{\chi}_{1}^{ \pm}$pairs assuming $W$-boson-mediated decays, as shown in Figure 12 (right), greatly improving limits obtained in an earlier search at $8 \mathrm{TeV}$ [33]. For slepton-pair-mediated decays of $\tilde{\chi}_{1}^{ \pm} \tilde{\chi}_{1}^{\mp}$ production, the exclusion reached $m_{\tilde{\chi}_{1}^{ \pm}}<1 \mathrm{TeV}$, whereas for slepton-pair production masses up to $700 \mathrm{GeV}$ were excluded assuming three generations of mass-degenerate sleptons.
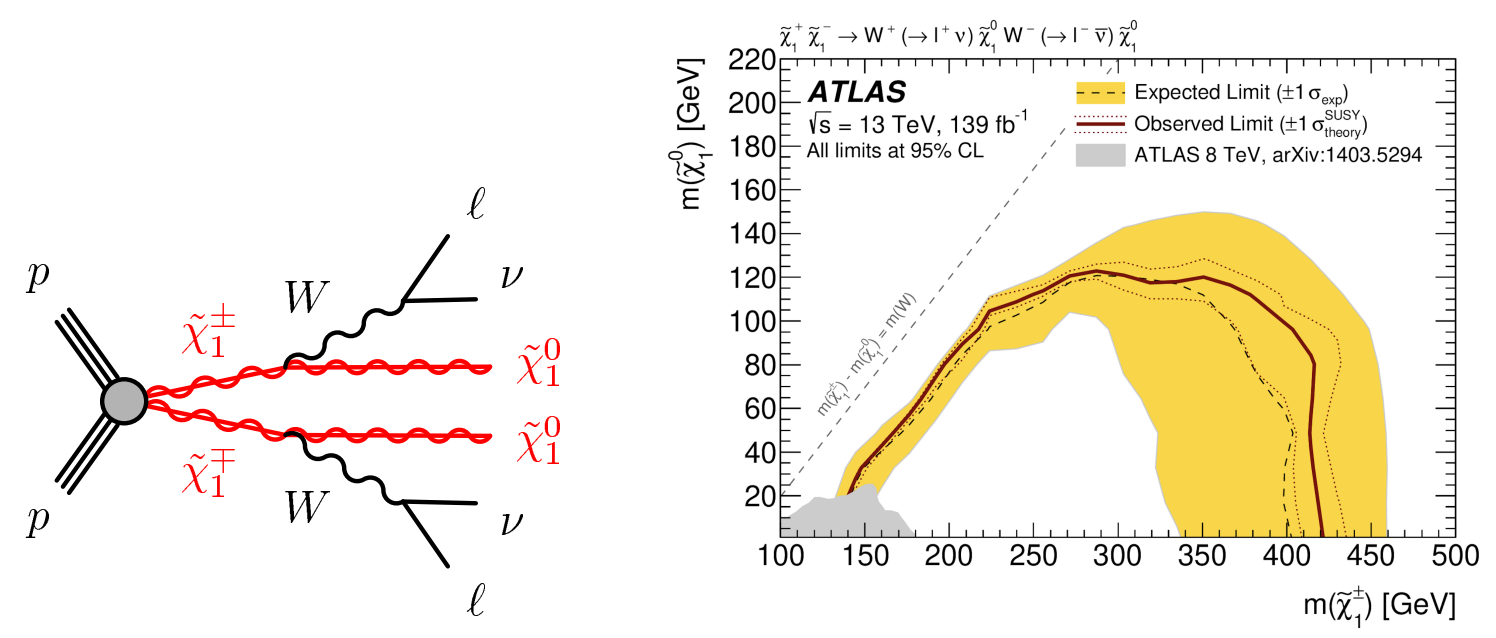

Figure 12: SUSY simplified model for chargino-pair production with $W$-boson-mediated decays. Left: Diagram of $\tilde{\chi}_{1}^{ \pm} \tilde{\chi}_{1}^{\mp}$ with $W$ mediation. Right: Observed and expected exclusion limits at $95 \%$ CL. The yellow band corresponds to the $\pm 1 \sigma$ variations in the expected limit. The dotted lines around the observed limit show the signal cross-section theoretical uncertainty. From [32].

\subsection{Compressed spectra}

Extensions of the SM that include new states with nearly degenerate masses can help to resolve open issues in particle physics while evading constraints from experiments at high-energy colliders. The mass spectra of such new states are referred to in the context of supersymmetric models as compressed. If produced in high energy collisions, such events would be characterised by the presence in the final state of low-momentum 'soft' leptons and/or jets. Therefore, triggering of such events cannot rely on, e.g., isolated high- $p_{\mathrm{T}}$ leptons and other strategies have been developed 
and applied by ATLAS and CMS to design the pertinent online and offline selections. Large- $E_{\mathrm{T}}^{\text {miss }}$ triggers were mostly used to select such events.

The CMS search [34], based on $35.9 \mathrm{fb}^{-1}$ collected in 2016, utilised events in four different channels depending on the number and type of leptons: $0 \ell \mathrm{jj}, e \mathrm{jj}, \mu \mathrm{jj}$, and $\tau_{\mathrm{h}} \mathrm{jj}$, where $\tau_{\mathrm{h}}$ denotes a hadronically decaying $\tau$ lepton. This was the first search for SUSY in the vector-boson fusion (VBF) topology with single soft-lepton, $\ell$, final states. The VBF topology required two wellseparated jets that appear in opposite hemispheres, with large invariant mass $m_{\mathrm{jj}}$. The observed $m_{\mathrm{jj}}$ and the $m_{\mathrm{T}}\left(\ell, p_{\mathrm{T}}^{\text {miss }}\right)$ distributions did not reveal any evidence for new physics.

The results were used to exclude a range of $\tilde{\chi}_{1}^{ \pm}$and $\tilde{\chi}_{2}^{0}$ gaugino masses. For a compressed mass spectrum scenario, in which $\Delta m \equiv m\left(\tilde{\chi}_{1}^{ \pm}\right)-m\left(\tilde{\chi}_{1}^{0}\right)=1(30) \mathrm{GeV}$ and in which $\tilde{\chi}_{1}^{ \pm}$and $\tilde{\chi}_{2}^{0}$ branching fractions to light sleptons are $100 \%, \tilde{\chi}_{1}^{ \pm}$and $\tilde{\chi}_{2}^{0}$ masses up to 112 (215) GeV were excluded at $95 \%$ CL [34]. The four channels were combined and the results are presented in Figure 13. For the scenario where the sleptons are too heavy and decays of the charginos and neutralinos proceed via $W$ and $Z$ bosons, $\tilde{\chi}_{1}^{ \pm}$and $\tilde{\chi}_{2}^{0}$ masses up to 112 (175) GeV were excluded at 95\% CL for $\Delta m=1(30) \mathrm{GeV}$.

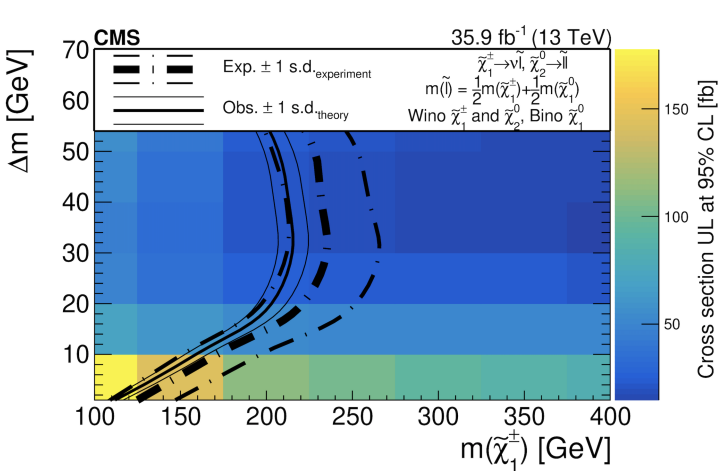

Figure 13: Expected and observed 95\% CL upper limit on the signal cross-section as a function of $m_{\tilde{\chi}_{1}^{ \pm}}$ and $\Delta m$, assuming the light slepton model with slepton mass defined as the average of the $\tilde{\chi}_{2}^{0}$ and $\tilde{\chi}_{1}^{ \pm}$ masses. From [34].

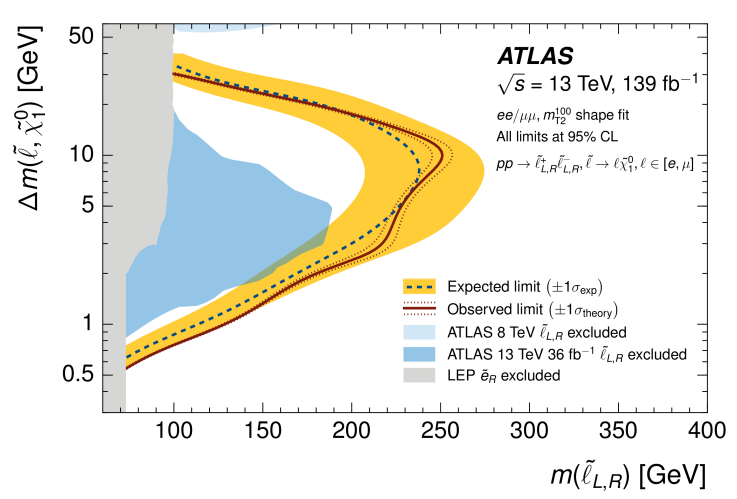

Figure 14: Expected 95\% CL sensitivity (dashed lines) and its $\pm 1 \sigma_{\exp }$ uncertainty (yellow band) for direct slepton production. The observed limit (red solid line) is shown with $\pm 1 \sigma_{\text {theory }}$ (dotted red line). From [35].

The most recent ATLAS search of this kind [35] was performed on the full Run 2 dataset of $139 \mathrm{fb}^{-1}$ by selecting events with $E_{\mathrm{T}}^{\text {miss }}$, two same-flavour, opposite-charge, low- $p_{\mathrm{T}}$ leptons, and hadronic activity from initial-state radiation or characteristic of vector-boson fusion production. The electrons or muons were required to have a minimum $p_{\mathrm{T}}$ of $5 \mathrm{GeV}$, whilst the corresponding requirement for the jet was $p_{\mathrm{T}}^{\mathrm{j}}>100 \mathrm{GeV}$.

The data were found to be consistent with SM predictions. The results were interpreted using simplified models of $R$-parity-conserving SUSY in which the LSP is the $\tilde{\chi}_{1}^{0}$ with a mass similar to the $\tilde{\chi}_{1}^{ \pm}$, the $\tilde{\chi}_{2}^{0}$, or the $\tilde{\ell}$. Lower limits on the masses of charginos in different simplified models ranged from 193 to $240 \mathrm{GeV}$ for moderate mass splittings, and extended down to mass splittings of 1.5 to $2.4 \mathrm{GeV}$ at the LEP chargino bounds $(92.4 \mathrm{GeV})$. Similar lower limits on degenerate light-flavor sleptons extended up to masses of $251 \mathrm{GeV}$ and down to mass splittings of $550 \mathrm{MeV}$, as shown in Figure 14. Slepton $\tilde{\ell}_{\mathrm{L}}\left(\tilde{\ell}_{\mathrm{R}}\right)$ refers to $\tilde{e}_{\mathrm{L}}$ and $\tilde{\mu}_{\mathrm{L}}\left(\tilde{e}_{\mathrm{R}}\right.$ and $\left.\tilde{\mu}_{\mathrm{R}}\right)$. The sleptons were 
assumed to be fourfold mass degenerate with $m_{\tilde{e}_{\mathrm{L}}}=m_{\tilde{e}_{\mathrm{R}}}=m_{\tilde{\mu}_{\mathrm{L}}}=m_{\tilde{\mu}_{\mathrm{R}}}$. Previous limits from earlier analyses $[33,36]$ are also shown. Constraints on vector-boson fusion production of electroweak SUSY states were also set [35].

CMS has also carried out a search for compressed SUSY involving soft $\tau$ leptons [37]. It is the first collider search with exactly one soft, hadronically decaying $\tau$ lepton and a large $E_{\mathrm{T}}^{\text {miss }}$ resulting from the recoil effect of a high transverse momentum jet from initial state radiation. The search utilised data corresponding to an integrated luminosity of $77.2 \mathrm{fb}^{-1}$ collected in 2016 and 2017. This particular search targeted compressed mass spectra where the mass difference between the $\tilde{\chi}_{1}^{ \pm}$, or the $\tilde{\chi}_{2}^{0}$, and the $\tilde{\chi}_{1}^{0}, \Delta m$, is $50 \mathrm{GeV}$. This choice was motivated by models considering $\tilde{\tau}-\tilde{\chi}_{1}^{0}$ co-annihilation aiming to maintain consistency in the estimation of the relic dark matter density between particle physics and cosmology. In the context of the MSSM, the search considered purely electroweak production of $\tilde{\tau}$ via cascading decays of $\tilde{\chi}_{1}^{ \pm}$and $\tilde{\chi}_{2}^{0}$ as well as direct production of $\tilde{\tau}$. The data did not reveal any evidence for new physics. The results were used to exclude a range of $\tilde{\chi}_{1}^{ \pm}$masses for a mass splitting $\Delta m\left(\tilde{\chi}_{1}^{ \pm}, \tilde{\chi}_{1}^{0}\right)$ of $50 \mathrm{GeV}$. For $\Delta m\left(\tilde{\chi}_{1}^{ \pm}, \tilde{\chi}_{1}^{0}\right)=50 \mathrm{GeV}$ and $\mathscr{B}\left(\tilde{\chi}_{1}^{ \pm} \rightarrow \tilde{\tau} v \rightarrow \tau \tilde{\chi}_{1}^{0} v\right)=100 \%, \tilde{\chi}_{1}^{ \pm}$masses up to $290 \mathrm{GeV}$ were excluded at $95 \% \mathrm{CL}$, as shown in Figure 15 . This sensitivity exceeded that of earlier $\tilde{\tau}$ searches [36, 38-40]

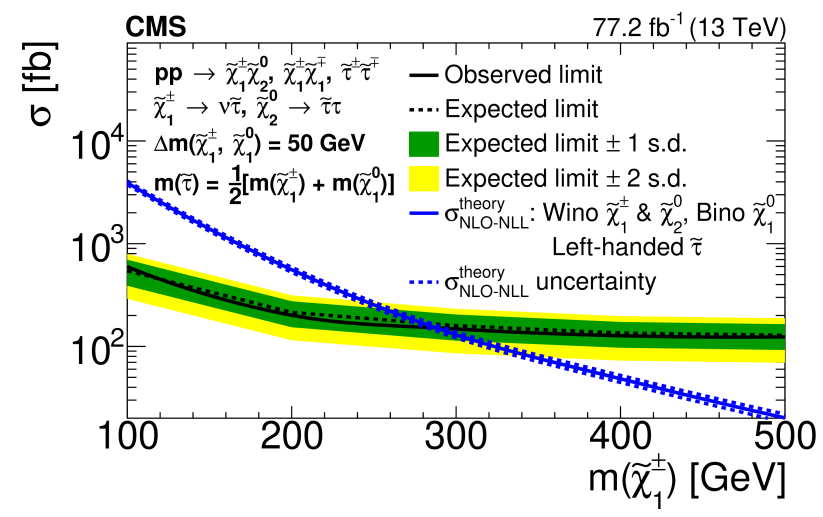

Figure 15: The 95\% CL observed (solid black line) and expected (dashed black line) upper limits on the SUSY production cross-sections as a function of $m\left(\tilde{\chi}_{1}^{ \pm}\right)$. The solid (dashed) blue line is the theoretical cross-section (its uncertainty). The green (yellow) band corresponds to the $\pm 1 \sigma( \pm 2 \sigma)$ range about the central value of the expected limit. From [37].

\section{5. $R$-parity violating SUSY}

$R$ parity is defined as $R=(-1)^{3(B-L)+2 S}$, where $B(L)$ is the baryon (lepton) number and $S$ the spin, respectively, granting $R=+1(R=-1)$ to all SM particles (SUSY partners) [41]. It is worth emphasising that the conservation of $R$ parity is merely an ad-hoc assumption with the only strict limitation coming from the proton lifetime: non-conservation of both $B$ and $L$ leads to a rapid proton decay. $R$-parity conservation has serious consequences in SUSY phenomenology in colliders: the SUSY particles are produced in pairs and, most importantly, the LSP is absolutely stable and weakly interacting, thus providing the characteristic high $E_{\mathrm{T}}^{\text {miss }}$ in SUSY events at colliders. An interesting feature of RPV SUSY is that it may be probed by looking for LSP resonances, something not possible in RPC due to the invisible LSPs. Besides that, if the RPV coupling is sufficiently small, the decay of the LSP into SM particles may be delayed, leading to distinctive signatures discussed, among other cases of long-lived particles, in Section 6. In view of the null results in conventional SUSY searches, it becomes necessary to fully explore non-standard SUSY scenarios also involving RPV and/or quasi-stable particles. 
Several of the searches conducted by ATLAS and CMS include SRs optimised for RPV scenarios, such as the ones requiring two same-sign leptons, highlighted in Section $2.2[16,20]$. The results of an earlier ATLAS SS analysis [19], together with those of other dedicated RPV analyses are projected onto the $\left(m_{\tilde{g}}, m_{\tilde{\chi}_{1}^{0}}\right)$ plane in Figure 16 [42]. The signal models assume the decay of the $\tilde{\chi}_{1}^{0}$ LSP into a charged lepton and two quarks, in the case of $\lambda^{\prime}$ coupling [19, 43], or three quarks, when a $\lambda^{\prime \prime}$ coupling is considered $[19,43,44]$.

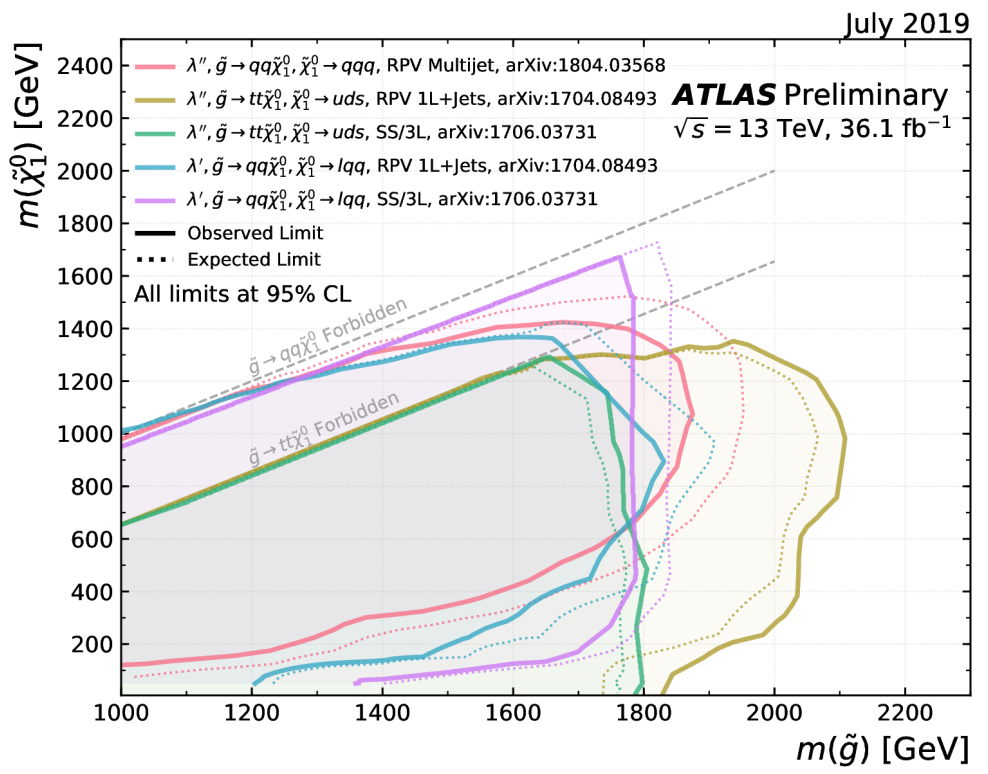

Figure 16: Exclusion limits at $95 \% \mathrm{CL}$ based on $13 \mathrm{TeV}$ data in the $\left(\tilde{g}, \tilde{\chi}_{1}^{0}\right)$ mass plane for different simplified models featuring the decay of the $\tilde{g}$ to the $\tilde{\chi}_{1}^{0}$ LSP which in turn decays via RPV couplings to SM particles. For each line, the $\tilde{g}$ decay mode is reported in the legend and it is assumed to proceed with $100 \%$ branching ratio. Some limits depend on additional assumptions. From [42].

Even if an analysis does not target RPV SUSY, its results may be reinterpreted in terms of suitable RPV models. Such reinterpretations have been carried out by ATLAS with $8 \mathrm{TeV}$ [45] and $13 \mathrm{TeV}$ searches [46]. The latter includes the reinterpretation of existing ATLAS analyses in models with variable $R$-parity coupling strength. Three simplified models targeting RPV scenarios with $\tilde{\chi}_{1}^{0} \rightarrow q q q$ through a $\lambda^{\prime \prime}$ coupling, were analysed. The large variations in final state, and therefore in sensitivity, as a function of the $R$-parity coupling strength motivate a thorough examination of the full ATLAS SUSY coverage. Different degrees of sensitivity were observed as a function of $\lambda^{\prime \prime}$. In the $\tilde{g}$ model with large branching fractions to top quarks, gluinos were excluded up to masses of $1.8 \mathrm{TeV}$, over the full range of lifetime and RPV coupling strengths. In the $\tilde{g}$ model with decays to first and second generation quarks, the differences are even more striking: for low values of the coupling, $\tilde{g}$ masses were excluded up to $2.0 \mathrm{TeV}$, but at high values of $\lambda^{\prime \prime}$ no limits were set [46].

The $\tilde{t}$ model is showcased in Figure 17, which exhibited large variations in the limits [46]. In the RPC regime, and for low values of $\lambda_{323}^{\prime \prime}$, the RPC stop 0L [47] and stop 1L [48] analyses set the strongest limits. The reliance on high $E_{\mathrm{T}}^{\text {miss }}$ signatures quickly reduced the sensitivity at even moderate values of $\lambda_{323}^{\prime \prime}$ and $\tilde{\chi}_{1}^{0}$ lifetime. The RPV $1 \mathrm{~L}$ analysis [43], in green, began setting limits for slightly higher values of $\lambda_{323}^{\prime \prime}$, setting its strongest limits around $\lambda^{\prime \prime} \simeq 10^{-2}$. The gap between 
the stop $0 \mathrm{~L} / 1 \mathrm{~L}$ and RPV $1 \mathrm{~L}$ analyses at $\lambda^{\prime \prime} \simeq 10^{-4}$, equivalent to $\tilde{\chi}_{1}^{0}$ lifetimes around $1 \mathrm{~ns}$, can potentially be closed by new searches utilising displaced vertices or displaced leptons. At very high values of $\lambda_{323}^{\prime \prime}$, the stop dijet pairs analysis [49], in black, placed limits for low values of the $\tilde{t}$ mass. The single stop resonant production, accessible via the dijet analysis [50] and Trigger-object Level Analysis (TLA) [51], set extremely strong limits on $\tilde{t}$ mass of $2.4 \mathrm{TeV}$ for high values of $\lambda^{\prime \prime} \simeq 1$.

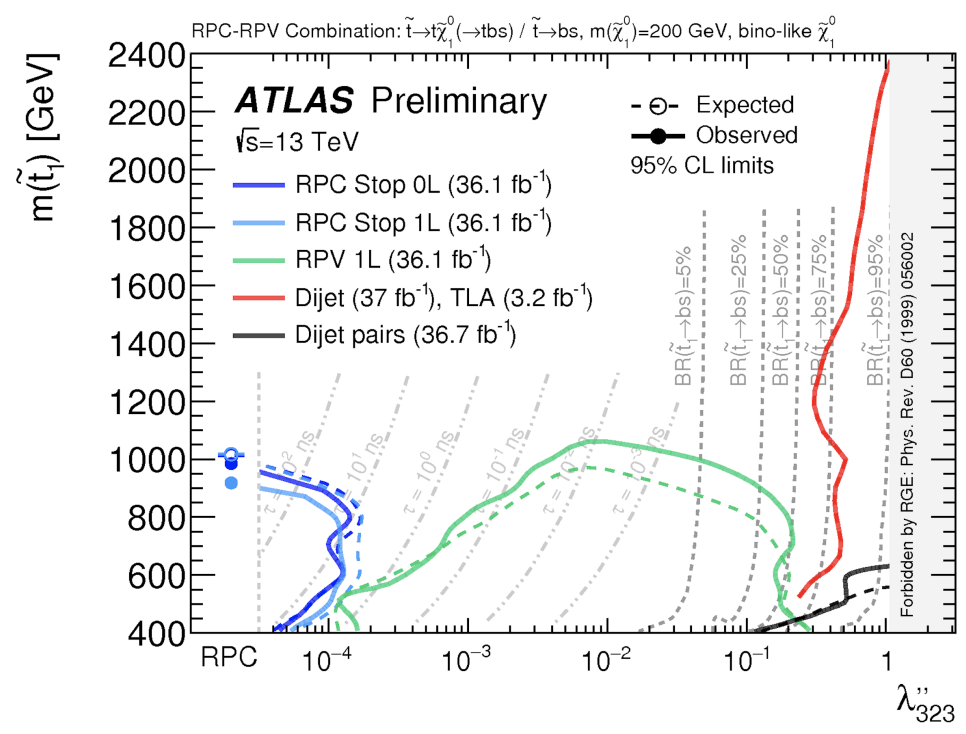

Figure 17: Observed (solid lines) and expected (dashed lines) exclusion limits for the $\tilde{t}$ model as a function of $\lambda_{323}^{\prime \prime}$ and $m_{\tilde{t}_{1}}$. The RPC-limit is shown on the leftmost part of the axes, while the region $\lambda_{323}^{\prime \prime}>1.07$ is forbidden by constraints from the renormalisation group equations. No expected limit is shown for the dijet and TLA results. From [46].

\section{Long-lived particles}

In some supersymmetric theoretical scenarios, the existence of long-lived particles (LLPs) is predicted [52], that may either decay within the typical volume of an LHC detector or may traverse it entirely as (meta)stable. In the former case, it may give rise to displaced vertices [5358], disappearing tracks $[12,59,60]$ or other signatures. On the other hand, heavy stable charged particles can be probed though the anomalous ionisation that they give rise to [61, 62]. The generalpurpose ATLAS and CMS experiments at the LHC are searching for and have set limits in LLP scenarios $[63,64]$. Besides them, dedicated detectors are being proposed to explore these lessconstrained manifestations of BSM physics [64].

\subsection{Displaced vertices}

A search for long-lived particles decaying into an oppositely charged lepton pair, $\mu \mu, e e$, or $e \mu$, was carried out by ATLAS using $32.8 \mathrm{fb}^{-1}$ of $p p$ collision data at $13 \mathrm{TeV}$ [57]. Candidate leptons were required to form a vertex, within the inner tracking volume of ATLAS, displaced from the primary $p p$ interaction region. The standard tracking requirements on the impact parameters, 
calculated relative to the beamline and beam spot, limit the reconstruction efficiency for highly displaced decays of LLPs. In order to improve the efficiency, an LLP-optimised track reconstruction algorithm, large radius tracking [65], as well as a dedicated displaced-vertex reconstruction were used.

No lepton pairs with an invariant mass greater than $12 \mathrm{GeV}$ were observed, consistent with the background expectations derived from data. The result was interpreted in the simplified model shown in Figure 18 (left), where the $\tilde{\chi}_{1}^{0} \mathrm{LSP}$, produced via $\tilde{q} \overline{\tilde{q}}$ production, decays into $\ell^{+} \ell^{\prime-} v$, where $\ell^{+}, \ell^{\prime-}=e, \mu$ with a finite lifetime due to the presence of $\lambda$-type RPV couplings. Crosssection limits were obtained for specific $\tilde{q}$ and $\tilde{\chi}_{1}^{0}$ masses. For a $700 \mathrm{GeV} \tilde{q}$, neutralinos with masses of $50-500 \mathrm{GeV}$ and mean proper lifetimes corresponding to $c \tau$ values between $1 \mathrm{~mm}$ to $6 \mathrm{~m}$ are excluded [57]. For a $1.6 \mathrm{TeV} \tilde{q}, c \tau$ values between $3 \mathrm{~mm}$ to $1 \mathrm{~m}$ were excluded for $1.3 \mathrm{TeV}$ neutralinos, as shown in Figure 18 (right). Similar results for $\tilde{g}$ pair production are presented in Ref. [53].
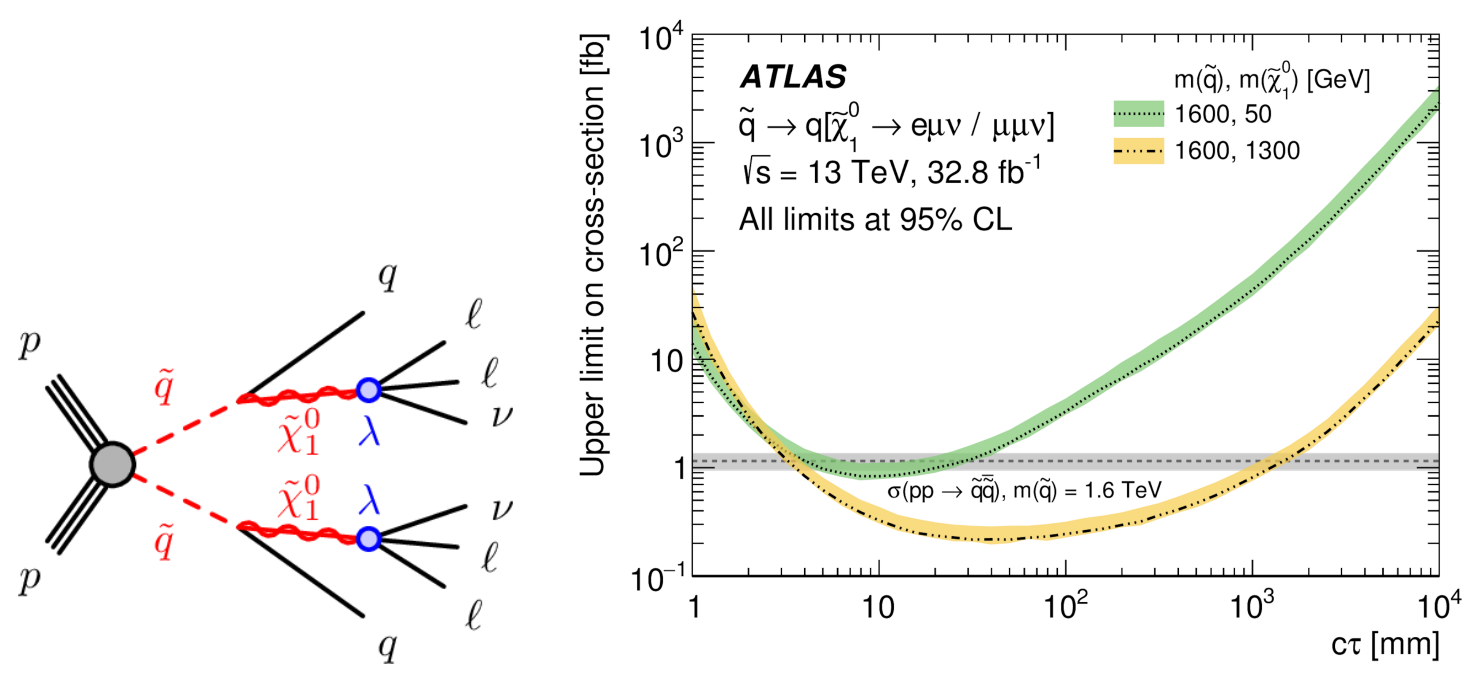

Figure 18: Diagram for the RPV SUSY model, where the decay of the $\tilde{\chi}_{1}^{0}$ is displaced from the $p p$ collision. Observed upper limits at $95 \% \mathrm{CL}$ on the squark-antisquark production cross-section as a function of the mean proper lifetime of the $\tilde{\chi}_{1}^{0}$, in units of $c \tau$, for the $\lambda_{122}$ scenarios and a $1.6 \mathrm{TeV}$ squark. The horizontal lines indicate the theoretical cross-sections with the uncertainties shown as shaded bands. The shaded bands around the observed limits indicate the $\pm 1 \sigma$ variations in the expected limit. From [57].

\subsection{Delayed jets}

The CMS experiment performed an inclusive search for LLPs decaying to displaced, nonprompt jets and missing transverse momentum based on a data sample of $137 \mathrm{fb}^{-1}$ at $13 \mathrm{TeV}$ [58]. The search used the timing of energy deposits in the electromagnetic calorimeter to select delayed jets from the decays of heavy long-lived particles. Residual backgrounds, originating from beam halo and cosmic-ray muons and from core and satellite bunch collisions, were estimated using measurements in control regions in the data. The timing at event level was defined by the jet in the event with the largest $t_{\text {jet }}$ passing the relevant selection. No events were observed in data for $t_{\text {jet }}>3$ ns, i.e. in the signal region, as evident from Figure 19. 


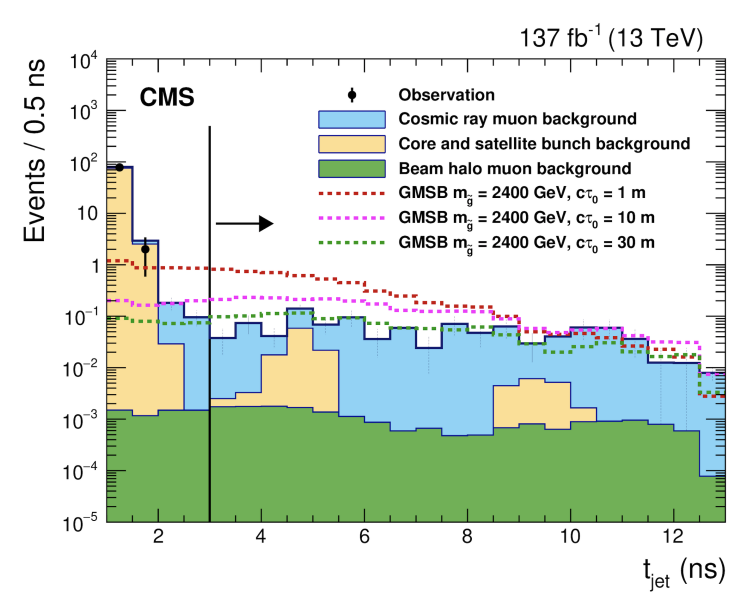

Figure 19: Timing distributions of the background sources, compared to those for representative signal models (dashed lines). The observed data is shown by the black points. The vertical black line indicates the SR definition. From [58].

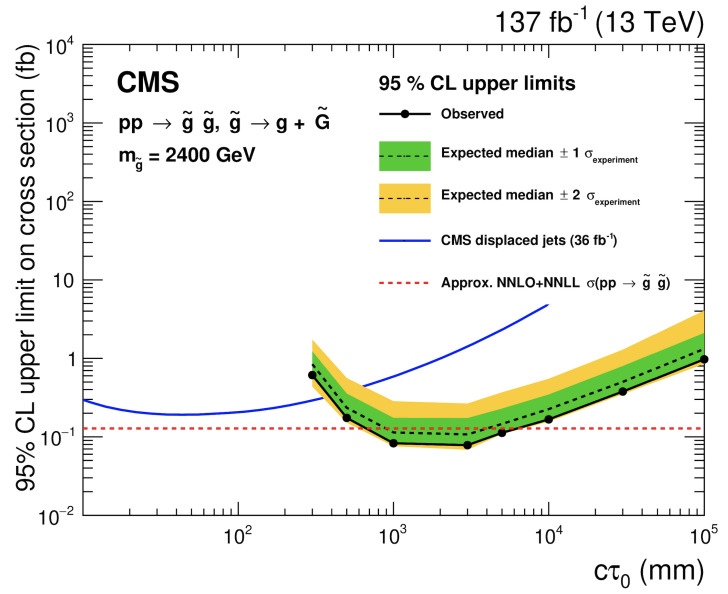

Figure 20: 95\% CL observed and expected (with $\pm 1 \sigma$ and $\pm 2 \sigma$ variations) upper limits on the $\tilde{g} \tilde{g}$ production cross-section for a $\tilde{g}$ GMSB model with $m_{\tilde{g}}=2.4 \mathrm{TeV}$. The blue line is the limit from the displaced jet search [55]. From [58].

The results were interpreted in the gluino gauge-mediated supersymmetry breaking (GMSB) signal model and $\tilde{g}$ masses up to 2100,2500 , and $1900 \mathrm{GeV}$ were excluded at $95 \%$ CL for proper decay lengths of $0.3,1$, and $100 \mathrm{~m}$, respectively [58]. The reach for models that predict significant $E_{\mathrm{T}}^{\text {miss }}$ in the final state was significantly extended beyond all previous searches [53, 55, 56], for proper decay lengths greater than $0.5 \mathrm{~m}$, as demonstrated in Figure 20.

\section{Summary}

Supersymmetry signals have been extensively sought after by the ATLAS and CMS experiments, motivated by various theoretical approaches, models and topologies: strong production, $3^{\text {rd }}$-generation fermions, electroweak production, $R$-parity violation, delayed decays among others. They lead to a wide spectrum of signatures: $E_{\mathrm{T}}^{\text {miss }}+$ jets + leptons / photons / $b$-jets $/ \tau$-leptons, displaced vertices, and it is not possible to cover all of them here. No significant deviation from known SM processes has been observed up to now with the data collected during LHC Run 2 at $\sqrt{s}=13 \mathrm{TeV}$. As both experimental techniques and search strategies keep evolving, ATLAS and CMS will keep looking for supersymmetry with the new data that become available at the LHC. Summary plots of most recent results are continuously updated in Refs. [66, 67].

\section{Acknowledgments}

The author would like to thank the Corfu2019 organisers for the opportunity to present this talk. This work was supported in part by the Generalitat Valenciana via the Project PROMETEOII/2017/033 and by the Spanish MICIU / AEI and the European Union / FEDER via the grant PGC2018-094856-B-I00. 


\section{References}

[1] J. Wess and B. Zumino, Supergauge Transformations in Four-Dimensions, Nucl. Phys. B 70 (1974) 39.

[2] A. Salam and J. A. Strathdee, Supersymmetry and Nonabelian Gauges, Phys. Lett. B 51 (1974) 353.

[3] S. Ferrara and B. Zumino, Supergauge Invariant Yang-Mills Theories, Nucl. Phys. B 79 (1974) 413.

[4] S. P. Martin, A Supersymmetry Primer, Adv. Ser. Direct. High Energy Phys. 18 (1998) 1 [hep-ph/9709356].

[5] W. Abdallah et al., Reinterpretation of LHC Results for New Physics: Status and Recommendations after Run 2, 2003.07868.

[6] ATLAS collaboration, The ATLAS Experiment at the CERN Large Hadron Collider, JINST 3 (2008) S08003.

[7] CMS collaboration, The CMS Experiment at the CERN LHC, JINST 3 (2008) S08004.

[8] L. Evans and P. Bryant, LHC Machine, JINST 3 (2008) S08001.

[9] ATLAS collaboration, Search for squarks and gluinos in final states with jets and missing transverse momentum using $36 \mathrm{fb}^{-1}$ of $\sqrt{\mathrm{s}}=13 \mathrm{TeV}$ pp collision data with the ATLAS detector, Phys. Rev. D97 (2018) 112001 [1712.02332].

[10] CMS collaboration, Inclusive search for supersymmetry in pp collisions at $\sqrt{s}=13$ TeV using razor variables and boosted object identification in zero and one lepton final states, JHEP 03 (2019) 031 [1812.06302].

[11] CMS collaboration, Search for supersymmetry in proton-proton collisions at $13 \mathrm{TeV}$ in final states with jets and missing transverse momentum, JHEP 10 (2019) 244 [1908 . 04722 ].

[12] CMS collaboration, Searches for physics beyond the standard model with the $M_{\mathrm{T} 2}$ variable in hadronic final states with and without disappearing tracks in proton-proton collisions at $\sqrt{s}=13$ TeV, Eur. Phys. J. C80 (2020) 3 [1909.03460].

[13] C. G. Lester and D. J. Summers, Measuring masses of semiinvisibly decaying particles pair produced at hadron colliders, Phys. Lett. B463 (1999) 99 [hep-ph/9906349].

[14] ATLAS collaboration, Search for squarks and gluinos in final states with jets and missing transverse momentum using $139 \mathrm{fb}^{-1}$ of $\sqrt{\mathrm{s}}=13 \mathrm{TeV}$ pp collision data with the ATLAS detector, ATLAS-CONF-2019-040, http://cds.cern.ch/record/2686254, 2019.

[15] P. Speckmayer, A. Hocker, J. Stelzer and H. Voss, The toolkit for multivariate data analysis, TMVA 4, J. Phys. Conf. Ser. 219 (2010) 032057.

[16] CMS collaboration, Search for physics beyond the standard model in events with jets and two same-sign or at least three charged leptons in proton-proton collisions at $\sqrt{s}=13 \mathrm{TeV}$, 2001.10086.

[17] CMS collaboration, Search for physics beyond the standard model in events with two leptons of same sign, missing transverse momentum, and jets in proton-proton collisions at $\sqrt{s}=13 \mathrm{TeV}$, Eur. Phys. J. C77 (2017) 578 [1704.07323].

[18] CMS collaboration, Search for supersymmetry in events with at least three electrons or muons, jets, and missing transverse momentum in proton-proton collisions at $\sqrt{s}=13 \mathrm{TeV}, \mathrm{JHEP} 02$ (2018) 067 [1710.09154]. 
[19] ATLAS collaboration, Search for supersymmetry in final states with two same-sign or three leptons and jets using $36 \mathrm{fb}^{-1}$ of $\sqrt{\mathrm{s}}=13 \mathrm{TeV}$ pp collision data with the ATLAS detector, JHEP 09 (2017) $084[1706.03731]$.

[20] ATLAS collaboration, Search for squarks and gluinos in final states with same-sign leptons and jets using $139 \mathrm{fb}^{-1}$ of data collected with the ATLAS detector, 1909.08457.

[21] R. Barbieri and G. F. Giudice, Upper Bounds on Supersymmetric Particle Masses, Nucl. Phys. B306 (1988) 63.

[22] B. de Carlos and J. A. Casas, One loop analysis of the electroweak breaking in supersymmetric models and the fine tuning problem, Phys. Lett. B309 (1993) 320 [hep-ph/9303291].

[23] CMS collaboration, Search for direct top squark pair production in events with one lepton, jets, and missing transverse momentum at $13 \mathrm{TeV}$ with the CMS experiment, 1912.08887.

[24] CMS collaboration, Measurement of the cross section for top quark pair production in association with a W or Z boson in proton-proton collisions at $\sqrt{s}=13 \mathrm{TeV}, \mathrm{JHEP} 08$ (2018) 011 [1711.02547].

[25] CMS collaboration, Searches for pair production of third-generation squarks in $\sqrt{s}=13 \mathrm{TeV} p p$ collisions, Eur. Phys. J. C77 (2017) 327 [1612.03877].

[26] ATLAS collaboration, Search for bottom-squark pair production with the ATLAS detector in final states containing Higgs bosons, b-jets and missing transverse momentum, JHEP 12 (2019) 060 [1908.03122].

[27] M. Escudero, A. Berlin, D. Hooper and M.-X. Lin, Toward (Finally!) Ruling Out Z and Higgs Mediated Dark Matter Models, JCAP 1612 (2016) 029 [1609.09079].

[28] ATLAS collaboration, ATLAS Run 1 searches for direct pair production of third-generation squarks at the Large Hadron Collider, Eur. Phys. J. C75 (2015) 510 [1506. 08616 ].

[29] CMS collaboration, Search for supersymmetry using Higgs boson to diphoton decays at $\sqrt{s}=13 \mathrm{TeV}$, JHEP 11 (2019) 109 [1908.08500].

[30] ATLAS collaboration, Search for direct production of electroweakinos in final states with missing transverse energy and a Higgs boson decaying into photons in pp collisions at $\sqrt{s}=13$ TeV with the ATLAS detector, ATLAS-CONF-2019-019, http://cds.cern.ch/record/2676596, 2019.

[31] ATLAS collaboration, Search for chargino and neutralino production in final states with a Higgs boson and missing transverse momentum at $\sqrt{s}=13 \mathrm{TeV}$ with the ATLAS detector, Phys. Rev. D100 (2019) 012006 [1812.09432].

[32] ATLAS collaboration, Search for electroweak production of charginos and sleptons decaying into final states with two leptons and missing transverse momentum in $\sqrt{s}=13 \mathrm{TeV}$ pp collisions using the ATLAS detector, Eur. Phys. J. C80 (2020) 123 [1908.08215].

[33] ATLAS collaboration, Search for direct production of charginos, neutralinos and sleptons in final states with two leptons and missing transverse momentum in pp collisions at $\sqrt{s}=8$ TeV with the ATLAS detector, JHEP 05 (2014) 071 [1403.5294].

[34] CMS collaboration, Search for supersymmetry with a compressed mass spectrum in the vector boson fusion topology with 1-lepton and 0-lepton final states in proton-proton collisions at $\sqrt{s}=13 \mathrm{TeV}$, JHEP 08 (2019) 150 [1905.13059].

[35] ATLAS collaboration, Searches for electroweak production of supersymmetric particles with compressed mass spectra in $\sqrt{s}=13 \mathrm{TeV}$ pp collisions with the ATLAS detector, 1911.12606 . 
[36] ATLAS collaboration, Search for electroweak production of supersymmetric states in scenarios with compressed mass spectra at $\sqrt{s}=13$ TeV with the ATLAS detector, Phys. Rev. D97 (2018) 052010 [1712.08119].

[37] CMS collaboration, Search for supersymmetry with a compressed mass spectrum in events with a soft $\tau$ lepton, a highly energetic jet, and large missing transverse momentum in proton-proton collisions at $\sqrt{s}=13$ TeV, Phys. Rev. Lett. 124 (2020) 041803 [1910.01185].

[38] CMS collaboration, Combined search for electroweak production of charginos and neutralinos in proton-proton collisions at $\sqrt{s}=13 \mathrm{TeV}$, JHEP 03 (2018) 160 [1801.03957].

[39] CMS collaboration, Search for new physics in events with two soft oppositely charged leptons and missing transverse momentum in proton-proton collisions at $\sqrt{s}=13 \mathrm{TeV}$, Phys. Lett. B782 (2018) $440[1801.01846]$.

[40] ATLAS collaboration, Search for the direct production of charginos and neutralinos in final states with tau leptons in $\sqrt{s}=13 \mathrm{TeV}$ pp collisions with the ATLAS detector, Eur. Phys. J. C78 (2018) 154 [1708.07875].

[41] H. K. Dreiner, An Introduction to explicit R-parity violation, Adv. Ser. Direct. High Energy Phys. 21 (2010) 565 [hep-ph/9707435].

[42] ATLAS collaboration, SUSY July 2019 Summary Plot Update, ATL-PHYS-PUB-2019-022, https://atlas.web.cern.ch/Atlas/GROUPS/PHYSICS/PUBNOTES/ATL-PHYS-PUB-2019-022/, 2019.

[43] ATLAS collaboration, Search for new phenomena in a lepton plus high jet multiplicity final state with the ATLAS experiment using $\sqrt{s}=13$ TeV proton-proton collision data, JHEP 09 (2017) 088 [1704.08493].

[44] ATLAS collaboration, Search for R-parity-violating supersymmetric particles in multi-jet final states produced in pp collisions at $\sqrt{s}=13 \mathrm{TeV}$ using the ATLAS detector at the LHC, Phys. Lett. $\mathbf{B 7 8 5}$ (2018) 136 [1804.03568].

[45] ATLAS collaboration, Constraints on promptly decaying supersymmetric particles with lepton-number- and R-parity-violating interactions using Run-1 ATLAS data,

ATLAS-CONF-2015-018, http://cds.cern.ch/record/2017303, 2015.

[46] ATLAS collaboration, Reinterpretation of searches for supersymmetry in models with variable $R$-parity-violating coupling strength and long-lived R-hadrons, ATLAS-CONF-2018-003, http://cds.cern.ch/record/2308391, 2018.

[47] ATLAS collaboration, Search for a scalar partner of the top quark in the jets plus missing transverse momentum final state at $\sqrt{s}=13$ TeV with the ATLAS detector, JHEP 12 (2017) 085 [1709. 04183 ].

[48] ATLAS collaboration, Search for top-squark pair production in final states with one lepton, jets, and missing transverse momentum using $36 \mathrm{fb}^{-1}$ of $\sqrt{\mathrm{s}}=13 \mathrm{TeV}$ pp collision data with the ATLAS detector, JHEP 06 (2018) 108 [1711.11520].

[49] ATLAS collaboration, A search for pair-produced resonances in four-jet final states at $\sqrt{s}=13 \mathrm{TeV}$ with the ATLAS detector, Eur. Phys. J. C78 (2018) 250 [1710.07171].

[50] ATLAS collaboration, Search for new phenomena in dijet events using $37 \mathrm{fb}^{-1}$ of pp collision data collected at $\sqrt{s}=13 \mathrm{TeV}$ with the ATLAS detector, Phys. Rev. D96 (2017) 052004 [1703. 09127 ].

[51] ATLAS collaboration, Search for light dijet resonances with the ATLAS detector using a Trigger-Level Analysis in LHC pp collisions at $\sqrt{s}=13 \mathrm{TeV}$, ATLAS-CONF-2016-030, http://cds.cern.ch/record/2161135, 2016. 
[52] M. Fairbairn, A. C. Kraan, D. A. Milstead, T. Sjostrand, P. Z. Skands and T. Sloan, Stable massive particles at colliders, Phys. Rept. 438 (2007) 1 [hep-ph/ 0611040 ].

[53] ATLAS collaboration, Search for long-lived, massive particles in events with displaced vertices and missing transverse momentum in $\sqrt{s}=13 \mathrm{TeV}$ pp collisions with the ATLAS detector, Phys. Rev. D97 (2018) 052012 [1710.04901].

[54] ATLAS collaboration, Search for long-lived particles produced in pp collisions at $\sqrt{s}=13 \mathrm{TeV}$ that decay into displaced hadronic jets in the ATLAS muon spectrometer, Phys. Rev. D99 (2019) 052005 [1811.07370].

[55] CMS collaboration, Search for long-lived particles decaying into displaced jets in proton-proton collisions at $\sqrt{s}=13$ TeV, Phys. Rev. D99 (2019) 032011 [1811.07991].

[56] CMS collaboration, Search for long-lived particles with displaced vertices in multijet events in proton-proton collisions at $\sqrt{s}=13$ TeV, Phys. Rev. D98 (2018) 092011 [1808.03078].

[57] ATLAS collaboration, Search for displaced vertices of oppositely charged leptons from decays of long-lived particles in pp collisions at $\sqrt{s}=13 \mathrm{TeV}$ with the ATLAS detector, Phys. Lett. B801 (2020) 135114 [1907.10037].

[58] CMS collaboration, Search for long-lived particles using nonprompt jets and missing transverse momentum with proton-proton collisions at $\sqrt{s}=13 \mathrm{TeV}$, Phys. Lett. B797 (2019) 134876 [1906.06441].

[59] ATLAS collaboration, Search for long-lived charginos based on a disappearing-track signature in pp collisions at $\sqrt{s}=13 \mathrm{TeV}$ with the ATLAS detector, JHEP 06 (2018) 022 [1712.02118].

[60] CMS collaboration, Search for disappearing tracks as a signature of new long-lived particles in proton-proton collisions at $\sqrt{s}=13 \mathrm{TeV}, \mathrm{JHEP} 08$ (2018) 016 [1804.07321].

[61] CMS collaboration, Search for long-lived charged particles in proton-proton collisions at $\sqrt{s}=$ 13 TeV, Phys. Rev. D94 (2016) 112004 [1609.08382].

[62] ATLAS collaboration, Search for heavy charged long-lived particles in the ATLAS detector in $36.1 \mathrm{fb}^{-1}$ of proton-proton collision data at $\sqrt{s}=13 \mathrm{TeV}$, Phys. Rev. D99 (2019) 092007 [1902.01636].

[63] L. Lee, C. Ohm, A. Soffer and T.-T. Yu, Collider Searches for Long-Lived Particles Beyond the Standard Model, Prog. Part. Nucl. Phys. 106 (2019) 210 [1810.12602].

[64] J. Alimena et al., Searching for long-lived particles beyond the Standard Model at the Large Hadron Collider, 1903.04497.

[65] ATLAS collaboration, Performance of the reconstruction of large impact parameter tracks in the inner detector of ATLAS, ATL-PHYS-PUB-2017-014, http://cds.cern.ch/record/2275635, 2017.

[66] ATLAS Collaboration, "Summary plots from the ATLAS Supersymmetry physics group." https://atlas.web.cern.ch/Atlas/GROUPS/PHYSICS/CombinedSummaryPlots/SUSY/, 2018.

[67] CMS Collaboration, "CMS Exotica Summary plots for $13 \mathrm{TeV}$ data." https://twiki.cern.ch/twiki/bin/view/CMSPublic/SummaryPlotsEXO13TeV, 2018. 